\title{
Estudio de asociación por desequilibrio de ligamiento entre los genes TG FA, RARA, y BCL3 y fisura labiopalatina no sindrómica (FLPNS) en la población chilena
}

\author{
José Suazo $S^{1 a}$, José Luis Santos $M^{2 b}$, Viviana Silva $A^{1 c}$, Lilian Jara \\ $S^{1 d}$, Hernán Palomino $Z^{\text {le, }}$ Rafael Blanco $C^{1 e}$. \\ Possible association due to linkage \\ disequilibrium of TGFA, RARA and BCL3 \\ with nonsyndromic cleft lip with or without \\ cleft palate in the Chilean population
}

Background: Nonsyndromic cleft lip/palate (NSCLP) is a congenital malformation with the characteristics of a complex genetic trait. Based on experimental evidences as well as on association and linkage studies candidate genes TGFA, RARA and BCL3 have been postulated as being involved in the genetic etiology of this pathology. Aim: To test the possible association due to linkage disequilibrium between microsatellite markers located at less than $1 \mathrm{cM}$ from the three candidate genes and nonsyndromic cleft lip/palate using the case-parents trio design. Patients and Methods: The sample consisted of 58 case-parents trios. Two microsatellite markers, flanking each one of the candidate genes were analyzed by means of the polymerase chain reaction (PCR) with fluorescent labeled microsatellite markers. Electrophoresis of the PCR products was performed on a laser-fluorescent automatic DNA sequencer. Nonparametric ETDT was used to analyze the genotype data. Results: Significant linkage disequilibrium was detected between D2S443 (TGFA) and NSCLP. Significance was almost reached between D17S800 (RARA) and NSCLP. Alleles 239bp (D2S443) and 172bp (D17S800) showed significant preferential transmission from heterozygous parents to affected offspring. In the case of BCL3 both markers showed no significant results. Conclusions: The results of the present study do not show clear evidence that TGFA or RARA could be involved in the genetic etiology of NSCLP. Even though the importance of retinoic acid in the development of the embryo is well documented the results obtained for RARA are difficult to analyze. In relation to the possible role of BCL3 in NSCLP, recent information postulates that other genes located in the same chromosome region could be involved in NSCLP (Rev Méd Chile 2005; 133: 1051-58).

(Key Words: Cleft lip; Cleft palate; Linkage disequilibrium; Transforming growth factor alpha)

Recibido el 1 de diciembre, 2004. Aceptado el 19 de abril, 2005.

Trabajo parcialmente financiado por Proyecto FONDECYT 1011003.

${ }_{1}^{1}$ Programa de Genética Humana, Instituto de Ciencias Biomédicas, Facultad de Medicina, Universidad de Chile. ${ }^{2}$ Departamento de Epidemiología, Instituto de Nutrición y Tecnología de los Alimentos (INTA), Universidad de Chile.

aTecnólogo Médico, Programa de Doctorado en Ciencias Biomédicas, Fac. Medicina U. de Chile.

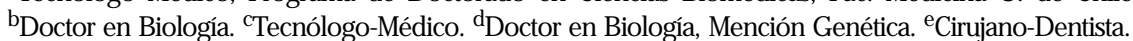

Correspondencia a: Dr. Rafael Blanco C. Av. Independencia 1027. Casilla 70061.

Teléfono: 56-2-6786457. Fax: 56-2-7373158. E mail: rblanco@med.uchile.cl 
L a fisura labiopalatina no sindrómica (FLPNS) es una de las malformaciones congénitas más frecuentes en el mundo. La naturaleza de la contribución genética en la etiología de la FLPNS es aún motivo de estudio y constituye un problema no resuelto, aunque también se han descrito influencias medioambientales en su desarrollo ${ }^{1}$.

En la actualidad el modelo de herencia más aceptado es el oligogénico, que propone que en el desarrollo de la FLPNS participarían varios genes interactuantes ${ }^{2}$. Ello concuerda con evidencias experimentales que demuestran que la morfogénesis del labio y paladar es el resultado de complejas interacciones celulares, procesos de migración, como es el caso de las células de la cresta neural, diferenciación y muerte celular, los cuales son controlados por una serie de proteínas estructurales y regulatorias ${ }^{3}$. Estos antecedentes permiten postular que los genes participantes en los procesos recién mencionados, constituyen posibles genes candidatos involucrados en la etiología genética de la FLPNS4.

En las últimas dos décadas se han realizado numerosos análisis genéticos de diversas regiones cromosómicas. Estudios de asociación y ligamiento, en poblaciones de origen caucásico, han sido en las que más frecuentemente se ha postulado la posible ubicación de genes candidatos en siete regiones, $2 p, 4 p, 4 q, 6 p, 14 q, 17 q$ y 19q $q^{5,6}$. Sin embargo, estudios similares en otros grupos étnicos, no han demostrado que estas mismas regiones cromosómicas estén involucradas en la FLPNS $^{7-9}$

El presente trabajo analiza tres regiones cromosómicas que no habían sido incluidas por nuestro grupo en publicaciones anteriores como parte de nuestra línea de investigación ${ }^{10-12}$. En el presente estudio se analiza el posible rol en la FLPNS de los genes candidatos Transforming Growth Factor Alpha (TGFA, 2p13.3), Retinoid Acid Receptor Alpha (RARA, 17q21.1) y B-cell leukemia/lymphoma-3 (BCL3, 19q13.1). Evidencias experimentales han postulado un rol de TGFA en la formación de los procesos palatinos ${ }^{13}$. Basados en estas evidencias, TGFA fue uno de los primeros genes en que se efectuaron estudios de asociación caso-control y de ligamiento paramétrico, los que mostraron resultados discordantes ${ }^{5,14-16}$. Recientemente, Moreno et $\mathrm{al}^{9}$ y Schultz et $\mathrm{al}^{8}$, comunican resultados positivos de ligamiento no paramétrico para este gen.
El segundo gen a analizar es el receptor del ácido retinoico alfa (RARA). El ácido retinoico, y su vía de señalización, están involucrados en el desarrollo normal de las estructuras frontonasales y maxilares ${ }^{17}$. Para este gen también se han realizado estudios de asociación caso-control y de ligamiento paramétricos, con resultados tanto positivos como negativos ${ }^{18,19}$. Otros autores, utilizando métodos no paramétricos, han reportado resultados negativos de asociación ${ }^{20}$, así como resultados positivos para ligamiento ${ }^{21}$.

El proto-oncogen BCL3 tendría un rol en la regulación transcripcional de genes involucrados en el ciclo celular ${ }^{22}$, y mutaciones en su secuencia podrían evitar la fusión de los primordios faciales $^{23}$. Basado en estas evidencias experimentales, Amos et $\mathrm{al}^{24}$ detectan desequilibrio de ligamiento entre un microsatélite de BCL3 y FLPNS, al igual que Martinelli et $\mathrm{al}^{25}$ y Gaspar et $\mathrm{al}^{4}$.

Los resultados mencionados en los párrafos precedentes, para TGFA, RARA y BCL3, al no ser concluyentes respecto al rol de estos genes en la etiología genética de la FLPNS, constituyen un antecedente que hace necesario continuar los análisis genéticos en poblaciones de distinto origen étnico, con métodos que eviten distorsiones en los resultados debido al efecto de la estratificación poblacional, dado que las diferencias observadas para los mismos genes candidatos pueden deberse a la heterogeneidad genética que muestra esta patología.

La variabilidad genética y étnica de la población chilena, es el resultado del proceso de mezcla entre la población nativa amerindia, de origen asiático, y los colonizadores españoles, de origen caucásico ${ }^{26}$. Esta característica de la población chilena, ofrece la oportunidad de analizar regiones cromosómicas en que se sostiene que se ubicarían posibles genes candidatos en poblaciones de diferente origen étnico.

El propósito del presente estudio es evaluar el posible desequilibrio de ligamiento entre marcadores de microsatélite de los genes TGFA, RARA y BCL3 con FLPNS, utilizando el diseño de tŕos caso-progenitores en una muestra de la población chilena.

\section{Materiales y MÉTodos}

Familias. La muestra de 58 tríos caso-progenitores fue obtenida del banco de datos genealógicos que 
posee el laboratorio de Genética Molecular Humana, ICBM, Facultad de Medicina, Universidad de Chile. Todos los tríos incluían al probando, los cuales no tenían relación de parentesco entre sí. Todas las familias seleccionadas tienen ancestros chilenos, sin inmigrantes e incluyendo pacientes que presentaban FLPNS como única enfermedad familiar. Además, las familias en que se constató el uso de drogas como warfarina, fenitoína o etanol, fueron excluidas del estudio. Los tríos casoprogenitores, provenientes de las genealogías mencionadas, pertenecen a los estratos socioeconómicos bajo a medio bajo, dada la composición genética de la población chilena, la relación existente entre etnicidad, marcadores genéticos de mezcla amerindia y estrato socioeconómico, mencionados en la introducción.

Análisis molecular. Después de obtener el consentimiento informado de todos los individuos que participaron en este estudio, consentimiento aprobado por la Comisión de Ética de la Facultad de Medicina, se procedió a tomar una muestra de $10 \mathrm{ml}$ de sangre periférica, de la cual se extrajo ADN genómico mediante el método descrito por Poncz et ${ }^{27}$.

En el presente estudio se utilizaron seis marcadores de microsatélite ubicados a menos de un centimorgan (cM) de los genes candidatos seleccionados; D2S292 y D2S443 para el gen TGFA, D17S800 y THRA-1 para el gen RARA, y BCL3-CA y D19S918 para BCL3. Mediante la reacción en cadena de la polimerasa (PCR) se amplificó el segmento de ADN correspondiente a estos seis microsatélites, utilizando los partidores descritos para D2S292 ${ }^{28}$, D2S44329, D17S80028, THRA-120, BCL3-CA ${ }^{30}$, y D19S412 ${ }^{28}$. Cada reacción se llevó a cabo según protocolo estándar de amplificación ${ }^{31}$, con las siguientes temperaturas de alineamiento: $53^{\circ} \mathrm{C}$ para $\mathrm{D} 17 \mathrm{~S} 800,54^{\circ} \mathrm{C}$ para THRA1, $55^{\circ} \mathrm{C}$ para D2S443, BCL3 y D2S292, y $64^{\circ} \mathrm{C}$ para D19S918. En este estudio se incorporó la utilización de fluorocromos para marcar el partidor directo (forward primer) lo que permite que los productos de la PCR sean analizados en un secuenciador automático, ABI Prism 310 de Applied Biosystem. Los resultados de la electroforesis fueron procesados mediante los programas computacionales GENESCAN 3.1.2 y Genotyper versión 2.5, que entrega las lecturas, en pares de bases, para los alelos de los distintos marcadores estudiados.
Estadística. Utilizando el diseño de tríos casoprogenitores, se aplicó el test de desequilibrio de transmisión multialélico (ETDT) ${ }^{32}$, para evaluar la posible asociación por desequilibrio de ligamiento entre los marcadores de microsatélite incluidos en este estudio y FLPNS. El desequilibrio de ligamiento (DL) se refiere a la asociación que existe entre alelos que se encuentran ubicados muy cercanamente en un mismo segmento cromosómico. El propósito de los estudios que relacionan genotipo y enfermedad es buscar asociación por DL entre un marcador genético anónimo (por ejemplo, un marcador microsatélite) y un gen de susceptibilidad, cuando existe cercanía física entre ambos. En el contexto de estudios de genotipoenfermedad, la asociación por DL surgiría como consecuencia de que ambos loci tenderían a heredarse conjuntamente y se mantendrían asociados en las personas enfermas, si no se han sucedido un número suficiente de generaciones que rompan esta asociación alélica. Debido a este tipo de asociación indirecta, la frecuencia de genotipos en el marcador anónimo será diferente en casos versus controles. En este estudio se utiliza el diseño de tríos caso-progenitores, que evita las asociaciones espurias de los estudios caso-control debidas a la estratificación genética por etnicidad de la población ${ }^{33}$. Si existe asociación por DL, la transmisión alélica observada en los tríos de caso-progenitores se desviaría de la probabilidad esperada (50\%) de la hipótesis nula de no asociación.

\section{RESULTADOS}

La Tabla 1 muestra los resultados globales de desequilibrio de ligamiento entre FLPNS y los marcadores de microsatélite estudiados, en un análisis de tipo alélico (allele-wise) de ETDT. De los marcadores de microsatélite que flanquean al gen TGFA, D2S443 mostró significancia estadística $(p=0,004)$, pero no así D2S292. En el caso de RARA, D17S800 casi alcanzó la significancia con un valor de $\mathrm{p}=0,07$ y THRA1 en cambio, no mostró resultados significativos. De los dos marcadores utilizados para el estudio de BCL3, uno intragénico (BCL3-CA) y uno extragénico (D19S412), ninguno mostró significación estadística.

La Tabla 2 presenta la transmisión preferencial individual de cada alelo del marcador D2S443 en 
Tabla 1. D esequilibrio de ligamiento entre marcadores de microsatélites flanqueadores de los genes T G FA (2p13.3), RARA (17q21.1) y BCL3 (19q13.1) y FLPN S utilizando el diseño de tríos caso-progenitores

\begin{tabular}{|cccc|}
\hline Gen & Marcador & № de tríos & $\mathrm{P}$ \\
\hline \multirow{2}{*}{ TGFA } & D2S292 & 51 & 0,65 \\
& D2S443 & 54 & 0,004 \\
RARA & D17S800 & 57 & 0,07 \\
& THRA-1 & 58 & 0,62 \\
BCL3 & D19S412 & 57 & 0,83 \\
& BCL3-CA & 49 & 0,56 \\
\hline
\end{tabular}

p: Valores de p sin corrección por comparaciones múltiples.

Tabla 2. Análisis del desequilibrio de la transmisión de los alelos del marcador D 25443 de progenitores heterocigotos a la progenie afectada

\begin{tabular}{|lccl|}
\hline Alelos (pb) & Transmitido & No transmitido & $\mathrm{P}$ \\
\hline$(215 \mathrm{pb})$ & 3 & 0 & - \\
$(219 \mathrm{pb})$ & 6 & 3 & - \\
$(223 \mathrm{pb})$ & 5 & 5 & 1 \\
$(227 \mathrm{pb})$ & 10 & 4 & 0,1 \\
$(231 \mathrm{pb})$ & 12 & 14 & 0,7 \\
$(235 \mathrm{pb})$ & 16 & 12 & 0,4 \\
$(239 \mathrm{pb})$ & 2 & 17 & 0,0006 \\
$(247 \mathrm{pb})$ & 9 & 6 & 0,4 \\
$(251 \mathrm{pb})$ & 3 & 4 & - \\
$(255 \mathrm{pb})$ & 0 & 1 & - \\
\hline
\end{tabular}

pb: Pares de bases. p: Valores de p sin corrección por comparaciones múltiples.

relación con el resto de los alelos de este polimorfismo, desde los progenitores heterocigotos a la progenie afectada. De todos los alelos analizados, sólo el alelo de 239 pb presentó una desviación significativa en su transmisión de progenitor a progenie $(p=0,0006)$. La Tabla 3 muestra el mismo análisis para el marcador D17S800 en la que se observa que sólo el alelo de $172 \mathrm{pb}$ alcanzó una significación estadística $(p=0,05)$, lo que indica también una desviación en la transmisión de este alelo de progenitor a progenie.
Tabla 3. Análisis del desequilibrio de la transmisión de los alelos de marcador D 175800 de los progenitores heterocigotos a la progenie afectada

\begin{tabular}{|lccl|}
\hline Alelos $(\mathrm{pb})$ & Transmitido & No transmitido & $\mathrm{p}$ \\
\hline$(164 \mathrm{pb})$ & 4 & 1 & - \\
$(166 \mathrm{pb})$ & 18 & 15 & 0,6 \\
$(168 \mathrm{pb})$ & 28 & 17 & 0,1 \\
$(170 \mathrm{pb})$ & 13 & 14 & 0,8 \\
$(172 \mathrm{pb})$ & 8 & 11 & 0,5 \\
$(174 \mathrm{pb})$ & 5 & 13 & 0,05 \\
$(176 \mathrm{pb})$ & 1 & 6 & - \\
\hline
\end{tabular}

pb: Pares de bases. p: Valores de p sin corrección por comparaciones múltiples.

\section{DisCUSIÓN}

El diseño de tríos caso-progenitores evita los problemas de resultados falsos positivos debidos a estratificación poblacional, básicamente porque el caso observado es siempre comparado con un spseudocontrol» pareado étnicamente (progenitores) ${ }^{34}$. Por lo tanto, dada la presencia de estratificación en la población chilena, este diseño parece ser el método más adecuado para detectar desequilibrio de ligamiento. Nuestro estudio utilizó 
una muestra de tríos caso-progenitores no relacionados, con el objetivo de detectar el posible desequilibrio de ligamiento entre marcadores de microsatélite, que flanquean los genes candidatos TGFA, RARA y BCL3, y la FLPNS.

Los resultados globales para ETDT muestran asociación significativa entre esta malformación y el marcador D2S443, ubicado a 0,1 cM centromérico a TGFA $(p=0,004)$. El análisis alelo por alelo de este polimorfismo muestra que de todos ellos sólo el alelo de 239 pares de bases (pb) presenta una desviación en su transmisión, de padres a progenie, que se aleja del $50 \%$ esperado $(p=0,0006)$. Este resultado, sumado al obtenido del análisis global de este marcador, refuerza el desequilibrio de ligamiento observado. Ello también podría implicar que uno de sus alelos en particular, parece transmitirse preferencialmente cuando segrega conjuntamente con el gen candidato, que portaría una mutación involucrada en la expresión de la FLPNS.

La existencia de desequilibrio de ligamiento entre el marcador D2S443 y la FLPNS, podían indicar que el gen TGFA estaría involucrado en la etiología genética de esta malformación. Sin embargo, llama la atención la ausencia de resultados significativos para D2S292, ubicado a 0,1 cM telomérico de este gen candidato. Dado que el tamaño muestral para ambos marcadores es idéntico, no es posible atribuir las diferencias observadas a este factor. Una posible explicación a estas diferencias obtenidas para ambos marcadores podrían reflejar que TGFA no sea el gen candidato en esta región cromosómica. Esta posibilidad se ve refrendada por los resultados comunicados por Pezzetti et al, en 199835, quienes reportan con resultados de ligamiento positivo para un marcador ubicado a $12 \mathrm{cM}$ de este mismo gen candidato. Estos autores postulan que no sería TGFA sino otro gen candidato ubicado en la misma región cromosómica. Otra posibilidad que no podemos descartar es que TGFA tenga sólo un efecto modificador más que ser un gen de fisura per se. Ello se sustenta en resultados experimentales en ratones mutantes nulos para TGFA que no presentan el fenotipo fisura ${ }^{36}$. Los resultados de los autores mencionados, sumados a aquellos obtenidos por nuestro estudio, demuestran que no se ha clarificado el rol que TGFA podría tener en la etiología genética de la FLPNS.
Los resultados globales de desequilibrio de ligamiento entre los marcadores de microsatélite que flanquean al gen RARA, muestran que D17S800 prácticamente alcanza la significancia estadística $(p=0,07)$, mientras que THRA1 no presenta resultados significativos $(p=0,62)$. Estos resultados llaman la atención ya que este marcador se ubica a 0,4 $\mathrm{cM}$ telomérico a este gen candidato y THRA1 se ubica a $0,2 \mathrm{cM}$ centromérico. El análisis alelo por alelo de D17S800 muestra que sólo el alelo de $174 \mathrm{pb}$ alcanza la significancia $(p=0,05)$. TRHA1 en cambio no presenta significancias para ningún alelo. Los resultados obtenidos para los marcadores de RARA se asemejan a aquellos previamente descritos para TGFA, lo que podría llevar a una conclusión similar, es decir, que otros genes en la región de RARA estarían involucrados en la expresión de la FLPNS. No obstante, el conocimiento sobre el rol del ácido retinoico y su vía de señalización en el desarrollo máxilo-facial ${ }^{17}$, hacen que los resultados obtenidos per se no permitan descartar la posible participación de RARA en la etiología genética de la FLPNS. Por otra parte debe considerarse que tanto los métodos paramétricos como no paramétricos presentan limitaciones en la detección de desequilibrio de ligamiento, cuando el gen candidato tenga sólo un efecto modificador en la expresión del rasgo en estudio ${ }^{37}$. Esta afirmación se ve refrendada por los discordantes resultados publicados por diversos autores en poblaciones de diferente origen étnico ${ }^{9,20}$.

En el caso del gen BCL3, ninguno de los marcadores utilizados, tanto el intragénico como el extragénico, presentaron significancia estadística tanto en el análisis global de desequilibrio de ligamiento, como en el análisis de la transmisión individual de los alelos de estos polimorfismos. Nuestros resultados no concuerdan con los publicados por algunos autores en diferentes poblaciones, quienes comunican asociaciones y ligamientos positivos, utilizando tanto métodos paramétricos como no paramétricos, pero concuerdan con aquellos publicados por Beaty et $\mathrm{al}^{4,16,25,34}$. La región cromosómica en que se ubica el locus para BCL3 ha sido repetidamente postulada como portadora de un gen candidato para FLPNS. Sin embargo, a la fecha, no existe evidencia que sustente un rol biológico de BCL3 en la patogénesis de esta 
malformación, a pesar de que se conoce su rol en la proliferación y muerte celular ${ }^{38}$. Esta aseveración se ve refrendada por los resultados de Yoshiura et $\mathrm{al}^{39}$, que describen una familia con FLPNS, en que cosegrega una translocación balanceada cuyo punto de ruptura en 19q interrumpe al gen TOMM40. Recientemente Suzuki ${ }^{40}$ observó que dos homólogos del gen PVRL1, PVR y PVRL2, localizados en 19q13.2 y flanqueado por BCL3, causan una forma sindrómica de fisura. Estos dos homólogos de PVRL1 junto con TOMM40 y BCL3 constituyen un cluster de genes. Estos antecedentes constituyen posible evidencia de que la cercanía de estos genes a BCL3 podría ser una posible causa de los resultados positivos de asociación y ligamiento comunicados para este último gen.

\section{REFERENCIAS}

1. Wyszynski DF, Beaty TH, Maestri NE. Genetic of nonsyndromic oral clefts revisited. Cleft Palate Craniofac J 1996; 33: 406-17.

2. FARRAL M, HoLder S. Familial recurrence-pattern analysis of cleft lip with or without clef palate. Am J Hum Genet 1992; 50: 270-7.

3. MurRay JC, Schutte BC. Cleft palate: players, pathways and pursuits. J Clin Invest 2004; 113: 1676-8.

4. Gaspar DA, Matioli SR, Pavanelo RC, Araújo BC, AndRÉ M, Steman S ET AL. Evidence that BCL3 plays a role in the etiology of nonsyndromic oral clefts in Brazilian families. Genetic Epidemiol 2002; 23: 364-74.

5. CARINCI F. Genetics of nonsyndromic cleft lip and palate: a review of international studies and data regarding the Italian population. Cleft Palate Craniofac J 2000; 37: 33-40.

6. MuRRAY JC. Gene/environment causes of cleft lip and/or palate. Clin Genet 2002; 61: 248-56.

7. Marazita ML, Field L, Cooper M, Tobias R, Maher B, Peanchitlertkajorn S, Lu Y. Genome scan for loci envolved in cleft lip with or without cleft palate, in Chinese multiplex families. Am J Hum Genet 2002; 71: 349-64.
En conclusión, los resultados del presente estudio concuerdan con aquellos postulados por diversos autores, en que no es posible concluir taxativamente, cuál sería el rol de TGFA en la expresión de la FLPNS. En el caso de RARA, el problema parece aún más difícil de dilucidar, pues los resultados de análisis genéticos, tanto de este estudio como de otros autores, no son concluyentes respecto a este gen, lo que se contrapone con el conocimiento del rol de este gen durante el desarrollo embrionario. Ello podría reflejar que mutaciones en RARA no tendrían un efecto de gen mayor en la expresión de esta patología. En cuanto a BCL3, los últimos hallazgos implican que es necesario analizar la zona en que este gen se ubica para dilucidar cuál de los genes que forman el cluster en que está BCL3, es realmente el gen involucrado en la FLPNS.

8. Schultz RE, Cooper ME, Daack-Hirsch S, Shi M, Nepomucena B, Graf KA et aL. Targeted scan of fifteen regions for nonsyndromic cleft lip and palate in Filipino families. Am J Med Genet 2004; 125A: $17-22$.

9. Moreno L, Arcos-Burgos M, Marazita M, Krahn K, MaHer B, CoOper M et al. Genetic analysis of candidate loci in non-syndromic cleft lip families from Antioquia-Colombia and Ohio. Am J Med Genet 2004; 125A: 135-44.

10. Bianco R, Chakraborty R, Carreño $H$, Paredes $M$, Palomino H, Barton $S$ et al. Evidence of a sexual dependent association between the MSX1 locus and nonsyndromic cleft lip with or without cleft palate in the Chilean population. Hum Biol 2001; 73: 81-9.

11. Bianco R, Suazo J, Santos JL, Carreño H, Paredes M, JARA L ET AL. Evaluación de la asociación entre marcadores de microsatélite en 6 p22-25 y fisura labiopalatina no sindrómica utilizando el diseño de tríos caso-progenitores en la población chilena. Rev Méd Chile 2003; 131: 765-72.

12. Blanco R, Suazo J, Santos JL, Carreño H, Palomino $\mathrm{H}$, JARA L No evidence for linkage and association between $4 \mathrm{q}$ microsatellite markers and nonsyndromic cleft lip palate in Chilean case-parents trios. Cleft Palate Craniofac J 2005 (in press). 
13. Pratt RM, Yoneda T, Silver MH, Salomon DS. Involvement of glucocorticoids and epidermal growth factor in secondary palate development. In: Pratt RM and Christiansen RL (eds) Current trends in prenatal craniofacial development. Elservier/North Holland, New York 1980; 235-52.

14. Ardinger HH, Burton KH, Bell GL, Bardach J, VanDemark DR, MurRaY JC. Association of genetic variation of the transforming growth factor alpha gene with cleft lip and palate. Am J Hum Genet 1989; 45: 348-53.

15. Jara L, Blanco R, Chiffele I, Palomino H, Carreño H. Association between alleles of the transforming growth factor alpha locus and cleft lip and palate in the Chilean population. Am J Hum Genet 1995; 57: 548-51.

16. Wyszynski DF, Maestri N, McIntosh I, Smith EA, García-Delgado C, Vinageras-Guarneros E et al. No evidence of linkage for cleft lip with or without cleft palate to a marker near the transforming growth factor alpha locus in two populations. Hum Hered 1997; 47: 101-9.

17. Helms JA, Kim CH, Hu D, Minkoff R, Thauer C, EICHEIE G. Sonic hedgehog participates in craniofacial morphogenesis and is down-regulated by teratogenic doses of retinoic acid. Dev Biol 1997; 187: 25-35.

18. CheneviX-Trench G, Jones K, Green AC, Duffy DL, MARTIN NG. Cleft lip with or without cleft palate: Associations with transforming growth factor alpha and retinoic acid receptor loci. Am J Hum Genet 1992; 51: 1377-85.

19. Shaw D, Ray A, Marazita M, Field Leigh. Further evidence of a relationship between the retinoic acid receptor alpha locus and nonsyndromic cleft lip with or without cleft palate (CL+P). Am J Hum Genet 1993; 53: 1156-7.

20. Kanno K, Suzuki Y, Yang X, Yamada A, Aoki Y, KURE $S$ ET AL. Lack of evidence for a significant association between nonsyndromic cleft lip with or without cleft palate and the retinoic acid receptor alpha gene in the Japanese population. J Hum Genet 2002; 47: 269-74.

21. Peanchitlertikajorn S, Cooper M, Liu Y, Field L, MaraziTA M. Chromosome 17: gene maping sudies of cleft lip with or without cleft plate in Chinese families. Cleft Pal Craniofac J 2003; 40: 71-9.

22. Fujita T, Nolan GP, Liou HC, Scott ML, Baltimore D. The candidate proto-oncogene BCL3 encodes a transcriptional coactivator that activates through
NF kappa B p50 homodimers. Genes Dev 1993; 7: 1354-63.

23. FERGUSON MW. Palate development: mechanisms and malformations. Ir J Med Sci 1987; 156: 309-15.

24. Amos C, Gasser D, Hecht JT. Nonsyndromic cleft lip with or without cleft palate: new BCL3 information. Am J Hum Genet 1996; 59:743-4.

25. Martinem M, Scapol L, Pezzetti F, Carinci F, Carinci P, BACILERO U ET AL. Suggestive linkage between markers on chromosome 19q13.2 and nonsyndromic orofacial cleft malformation. Genomics 1998; 51: 177-81.

26. Rothhammer F, Lasserre E, Bianco R, Covarrubias E, Dixon M. Microevolution in human Chilean populations. Shovel-shape, mesial palatal version and other dental traits in Pewenche indians. Z Morph Anthrop 1968; 162-9.

27. Poncz M, Solowiejcyk D, Harvel B, Mory Y, SCHWARTZ E, SURREY S. Construction of human gene libraries from small amounts of peripheral blood: analysis of beta-like globin genes. Hemoglobin 1982; 6: 27-36.

28. Gyapay G, Morissette J, Vignal A, Dib C, Fizames C, Milaseau $P$ ET AL. The 1993-94 généthon human genetic linkage map. Nature Genet 1994; 7: 246-9.

29. MurRaY JC. Face facts: genes, environment and clefts. Am J Hum Genet 1995; 57: 227-32. (Invited Editorial).

30. St George-Hyslop PH, Ohno H, Gusela JF, MckeIthan T. The BCL3 locus and chromosome 19 displays and informative microsatellite polymorphism. Nucleic Acid Research 1992; 20: 927.

31. Suazo J, Santos JL, Carreño H, Jara L, Blanco R. Linkage disequilibrium between MSX1 gene and nonsyndromic cleft lip palate in the Chilean population. J Dent Res 2004; 83: 782-5.

32. Sham PC, CurTis D. An extended transmission disequilibrium test for multi-allele marker loci. Ann Hum Genet 1995; 59: 323-36.

33. Cardon LR, Palmer LJ. Population stratification and spurious allelic association. Lancet 2003; 361: 598-604.

34. Beaty TH, Hetmanski JB, Zeiger JS, Fan YT, Lian KY, VANDERKOLK CA ET AL. Testing candidate genes for non-syndromic oral clefts using a case-parent trio design. Genetic Epidemiology 2002; 22: 1-11.

35. Pezzetti F, Scapoli L, Martinelu M, Carinci F, Bodo M, Carinci P et al. A locus in 2p13-p14 (OFC2), in addition to that mapped in $6 \mathrm{p} 23$, is involved in 
nonsyndromic familial orofacial cleft malformation. Genomics 1998; 50: 299-305.

36. Jugessur A, Lie RT, Wilcox AJ, MurRay JC, Taylor JA, SugSTAD OD ET AL. Variants of developmental genes (TGFA, TGFB3 and MSX1) and their associations with orofacial clefts: a case-parent triad analysis. Genet Epidemiol 2003; 24: 230-9.

37. GREENERG DA. Linkage analysis of mecessary» disease loci versus «susceptibility»loci. Am J Hum Genet 1993; 52: 135-43.

38. Cox TC. Taking it to the max: the genetic and developmental mechanisms coordinating midfa- cial morphogenesis and dysmorphology. Clin Genet 2004; 65: 163-76.

39. Yoshivra K, Machida J, Daack-Hirsch S, Patil SR, AsHworth LK, HeCht JT ET AL. Characterization of a novel gen disrupted by a balanced chromosomal translocation $t(2 ; 19)$ (q11.2; $q 13.3)$ in a family with cleft lip and palate. Genomics 1998; 54: 231-40.

40. Suzuki K, Hu D, Bustos T, Zlotogora J, RichieriCosta A, Helms JA ET al. Mutations of PVRL-1, encoding a cell-cell adhesion molecule/herpesvirus receptor, in cleft lip/palate-ectodermal dysplasia. Nature Genet 2000; 25: 427-30. 